\title{
LA CIUDAD DE LA HABANA Y SU MEDIO AMBIENTE
}

POR

NANCY PÉREZ RODRÍGUEZ

Antecedentes de la diferenciación ambiental en una ciudad

Los problemas ambientales son muy antiguos y complejos, surgen como resultado de la interacción de las sociedades humanas con el ambiente donde viven. Esta interacción ha tenido un largo desarrollo, ya que en la medida en que se hacen más complejas las relaciones en la sociedad, más intensidad adquiere la acción del hombre sobre la naturaleza y por tanto sobre su medio ambiente.

De hecho, desde un principio, la elección de una adecuada ubicación geográfica, que hace referencia a las condiciones naturales del espacio físico sobre el que se construye y organiza una ciudad, explican las razones que le dan lugar a su fundación, por lo que «Se puede afirmar que la influencia de los factores naturales en el origen y distribución de los primeros asentamientos era mucho mayor que en las ciudades actuales, ya que el control que podía ejercer el hombre sobre el medio era muy limitado» (12) *.

El proceso de urbanización conlleva a modificaciones de los elementos naturales base inicial de la diferenciación de los asentamientos humanos, provocando la creación de elementos artificiales que al combinarse e integrarse unos a los otros, originan la diferenciación

* Los números entre paréntesis se refieren a la Bibliografía al final. 
del ambiente urbano, mediatizados por los fundamentos técnicos y socioculturales de cada período o etapa histórica. «La naturaleza y la historia son dos elementos integrantes del mundo en que vivimos, nos movemos y nos manifestamos» (20)... «El urbanismo no viene impuesto a la naturaleza, sino que es añadido a ésta por el hombre para hacer más confortable su vida» (7).

El estudio del ambiente urbano como sistema complejo, plantea importantes retos conceptuales y metodológicos. Su materialización en la práctica aporta un conjunto de criterios muy necesarios para una mejor comprensión de lo que acontece en una ciudad.

El sistema urbano, abierto e interctivo, constituye la esencia del medio ambiente urbano, caracterizado por un aspecto natural o físico y otro sicosocial y todo ello referido a unas coordinadas históricas que dotan al concepto de una dimensión temporal y espacial. El medio ambiente, con una vertiginosa evolución en las últimas décadas, más de cien años después de su surgimiento, cargado de acepciones y de abordajes teórico-metodológicos, representa hoy tema de alta prioridad en los marcos académicos, investigativos y administrativopolíticos de cualquier nación del mundo.

Los nuevos enfoques permiten conceptualizar de forma sistémica e integral todo lo referente al ambiente, propiciando estrategias que contribuyan a la realidad. En tal sentido, se recurre a varios procedimientos que de una forma $u$ otra exploran la situación ambiental en el ámbito urbano, aunque no siempre se asuma con criterios integrales. Con tales referencias, el presente estudio se ha propuesto revelar las desigualdades del ambiente físico-social, en la Ciudad de La Habana, capital de la República de Cuba, apoyándose en un enfoque novedoso y arriesgado, con el interés de aproximarse a la conceptualización holística del medio ambiente urbano y la producción de un conocimiento sistematizado, dada las potencialidades de generalización y complementación de información.

\section{La Ciudad de La Habana. Aspectos de su heterogeneidad ambiental}

La Ciudad de La Habana se localiza en la costa norte de la región occidental de la isla caribeña, aproximadamente entre los 
$82^{\circ} 05^{\prime}$ y $83^{\circ} 35^{\prime}$ de longitud oeste, con una extensión territorial de $724 \mathrm{~km}^{2}$.

Entre sus accidentes geográficos más notables se encuentra la bahía de La Habana, típica bahía de bolsa en cuyas orillas empezó el desarrollo de la ciudad, extendiéndose luego hacia el sur y el oeste respectivamente (véase figura 1 ).

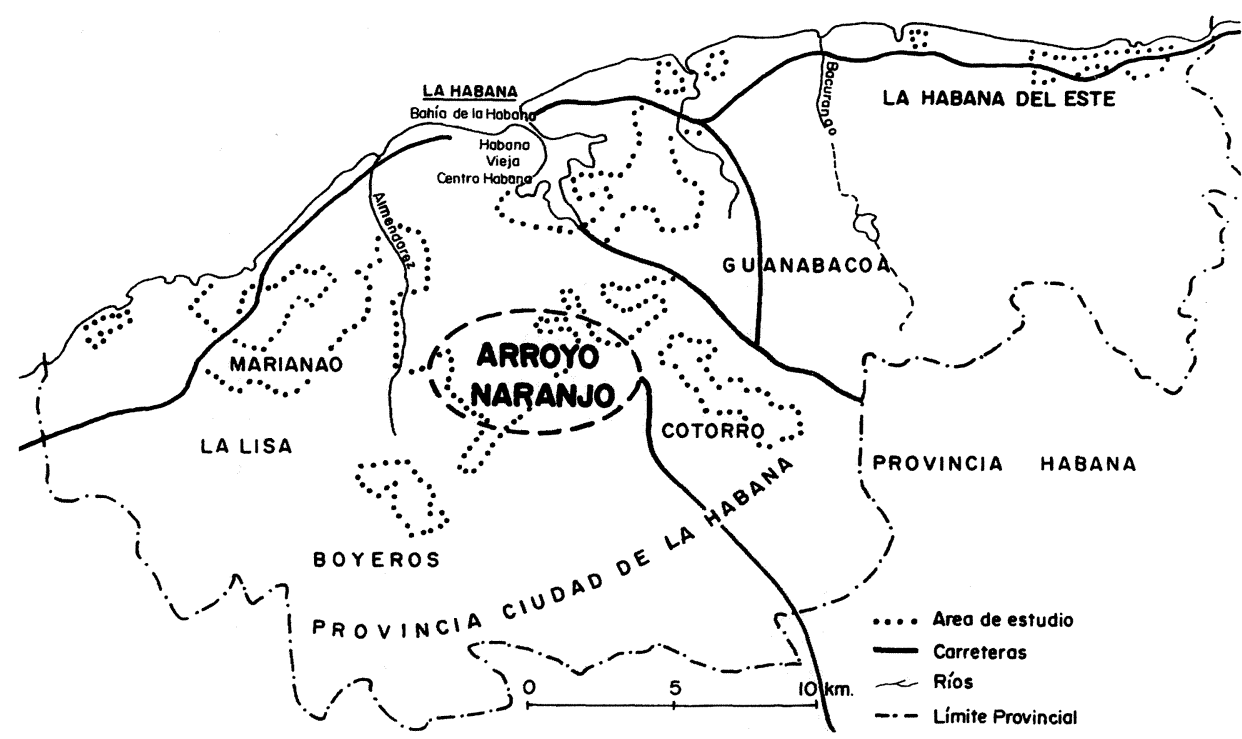

FIgURA 1.-Área de estudio.

Para comprender la forma en que se lleva a cabo la construcción del espacio en una ciudad, no basta sólo con explorar lo acontecido en los distintos períodos históricos transcurridos como consecuencia del desarrollo socioeconómico y del proceso de urbanización, dicho en otras palabras el espacio urbano como atributo de la sociedad.

Sin duda, el componente natural juega un papel decisivo como limitante o como factor que potencia o favorece el desarrollo de la actividad socioeconómica, es decir «el espacio efectivo fue creado a partir de la diferenciación ecológica». De ahí puede resultar un sinnúmero 
de espacios abiertos que por una u otra causa pueden retrasar el proceso de compactación de la ciudad por sólo citar un ejemplo.

Al hablar de las características de los componentes y paisajes naturales en La Habana hay que hacer notar su marcada diferenciación en el sentido latitudinal, que condiciona la diferenciación de fajas de llanuras casi paralelas a la línea de costa que tienen génesis y alturas diferentes. Lo anterior es reflejo de una relativa complejidad en su evolución paleogeográfica, relieve, así como suelos diferentes.

Por su parte, el clima, componente que menos varía en el contexto de la ciudad, se comporta en general como en el resto del territorio nacional, es decir tropical estacionalmente húmedo, con ligeras modificaciones por la influencia del mar, con lluvias frontales en invierno y convectivas en verano, aumentando de la costa hacia el interior. El régimen hidrológico está formado por 10 ríos que desembocan al mar en la vertiente norte y que corren en dirección norte-sur. Las áreas urbanizadas de la ciudad representan más del $40 \%$ del área total de la provincia de igual nombre, lo que da una idea de la ausencia casi total de vegetación natural.

Como resultado de la interacción de los componentes naturales, se identifican ocho complejos territoriales naturales de primer orden, treinta y cinco de segundo orden y trece de tercer orden (véase figura 2), que de conjunto la ubican en la región físico geográfica de la llanura norte de La Habana (16). Estas unidades han sido agrupadas según el orden en que aparecen dispuestas (N-S), aunque sólo se hará referencia a seis de ellas por ser las de mayores índices de urbanización dentro de la provincia.

Paisajes de las llanuras aterrazadas de 0-40 m, calcáreo marino, con rocas carbonatadas, rendzinas rojas y suelos rojos ferralíticos carbonatados, con bosque semideciduo en parte con vegetación de costa rocosa, arenosa y matorral xerofítico. Han sido distinguidas aquí 10 unidades de orden inferior que obedecen al grado y carácter de la karstificación en las superficies de las terrazas abrasivas, donde han influido notablemente las diversas fases litológicas y las peculiaridades del régimen neotectónico en relación directa con la dinámica litoral. 


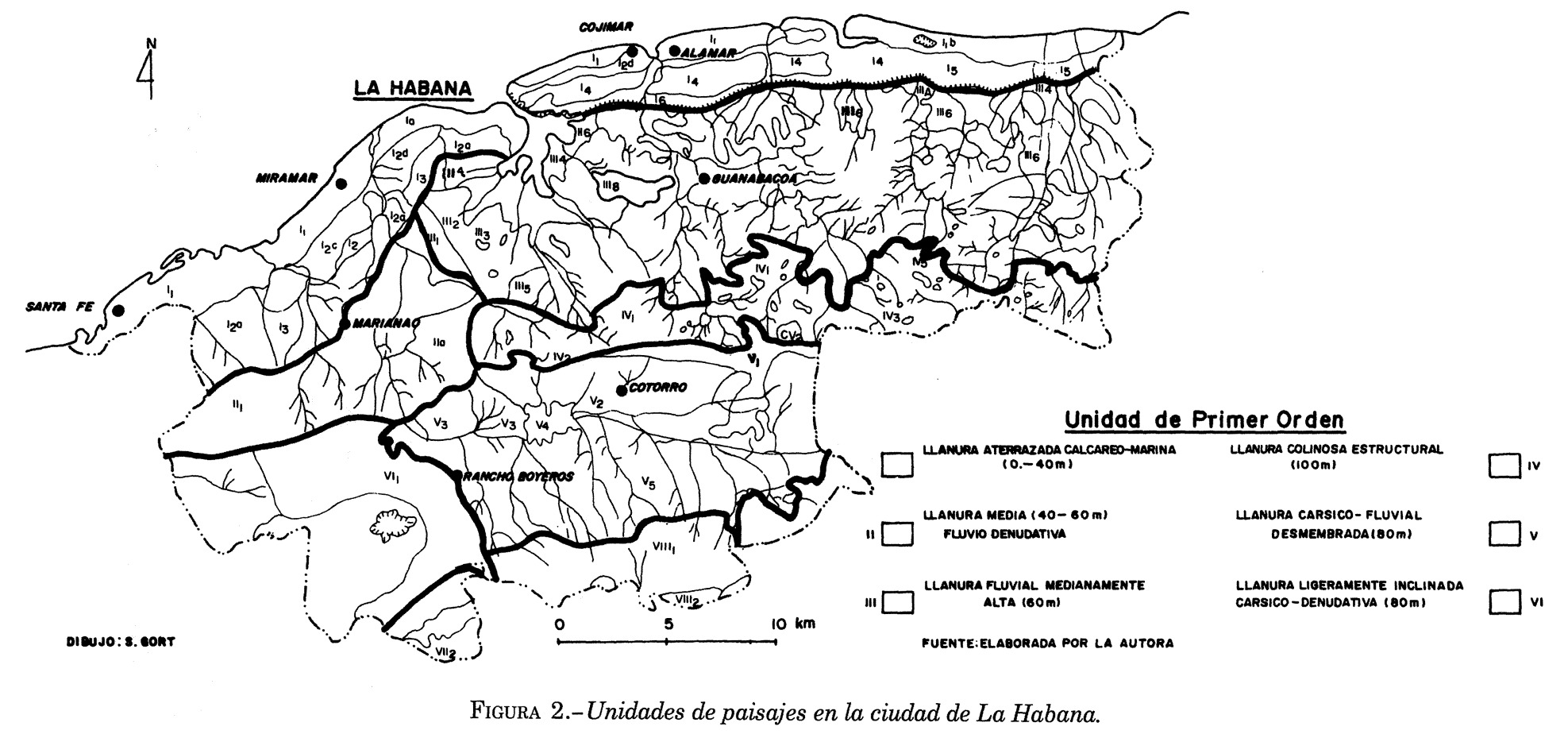


Paisajes de llanura fluvial $(40-60 \mathrm{~m})$ de altura con predominio de procesos denudativos, desarrollados sobre rocas terrígeno-carbonatadas, con vegetación herbácea, arbustiva y en parte con bosque semideciduo, sobre suelos pardos con o sin carbonatos. Donde lo más destacado es la acción erosiva de las corrientes fluviales de diversos órdenes. Se distinguen aquí el fondo de los valles de margas y flisch, inundables estacional y temporalmente, así como las colinas calcáreo-morgosas.

La tercera unidad es la llanura fluvial de hasta $60 \mathrm{~m}$ de altura, de ondulada o colinosa, erosivo-acumulativa, sobre calcarenitas y serpentinitas con bosque semideciduo y en parte matorral xerofítico sobre serpentinitas. Los procesos que han dado lugar a la formación de esta unidad obedecen en general a las etapas sucesivas de la evolución geólogo-geomorfológica, donde la erosión hizo desaparecer la cubierta de rocas carbonatadas preexistente, quedando la serpentinita al descubierto, más fácilmente atacable por la acción de los agentes del modelado, que dio lugar a una depresión a manera de valle longitudinal surcado por numerosas corrientes superficiales que la atraviesan en el sentido N-S y que han formado amplias superficies deltaicas en el borde septentrional como resultado del movimiento diferenciado de bloques. El mejor ejemplo es la Bahía de La Habana, «cuyos lóbulos o ensenadas constituían porciones de los valles inferiores de distintos ríos y arroyos, que se insertaban en un cauce común, convertido más tarde en el canal de entrada de la Bahía» (15).

La unidad de la llanura colinosa estructural de hasta $100 \mathrm{~m}$ de altura, sobre basamento de areniscas, flisch carbonatado, margas y en partes serpentinitas, con suelos pardos sialíticos carbonatados y ferromagnesianos, es la cuarta unidad distinguida de este estudio. Se introduce en el paisaje de la ciudad adoptando una forma de cuña con bordes redondeados, dando lugar a una serie de alturas que pueden llegar hasta los $100 \mathrm{~m}$, formado por estratos monoclinales que buzan al sur. Es considerada en parte aguas cristal de la provincia, siendo predominantes los procesos erosivos y denudativos.

La quinta localidad es la llanura cársico fluvial hasta $80 \mathrm{~m}$, desmembrada sobre calizas y depósitos aluviales, con bosque xerófilo y semideciduo sobre suelos pardos y rojos carbonatados. Desde el pun-

$$
-356-
$$


to de vista geomorfológico constituye un gran valle surcado por el río Almendares y su sistema de afluente con una dirección predominante este-oeste, en el que se destacan como procesos dominantes los provocados por la erosión fluvial, aunque hay manifestación de karst subsuperficial, lo que ha dado lugar a una extensa cuenca subterránea, conocida con el nombre de cuenca de Vento y que es una de las principales fuentes de abasto de agua de la ciudad.

La llanura ligeramente reclinada hasta $80 \mathrm{~m}$, cársico -denudativa sobre calizas y corteza de intemperismo rojo- amarillento con bosque mesófilo y semideciduo, sobre suelo pardo rojizo, ferralítico en parte fersialítico, representa una unidad con poco declive y alto grado de carsificación, siendo frecuente hallar depresiones cársicas de diversas dimensiones, así como el predominio del drenaje subsuperficial.

\section{Diferenciación del proceso de urbanización}

Fundada en 1519 «La ciudad posee una base geográfica que se hace visible desde los últimos años del siglo xvi (15). Como todo asentamiento humano y hasta convertirse en gran ciudad, no fue asimilada de forma continua. El motivo pudiera buscarse en un conjunto de factores: las de carácter natural (relieve e hidrografía), que decidieron su aspecto diferenciado en los inicios aportando las potencialidades para la futura urbanización y a los ya citados elementos de carácter socioeconómico que de hecho se convirtieron en los fundamentales una vez iniciado el proceso de expansión y desarrollo urbano.

Si se asocia el proceso de urbanización con las características naturales, podemos entender por qué las unidades con mayores potencialidades para el uso residencial no fueron ocupados tanto en tiempo como en espacio de igual manera, sino que por razones coyunturales de comunicación que llevan implícitas las posibilidades económicas, se observa un crecimiento a saltos predominando la dirección ya citada (oeste-sur) «Aunque la adaptación a la topografía se revela en la distribución de sus áreas urbanizadas» (15). En tal sentido, la diferenciación natural del territorio se integra en algunos casos a la explicación de la situación ambiental más favorable, dado por la ele-

$$
-357-
$$


vada potencialidad para la urbanización de algunos paisajes que coyunturalmente fueron urbanizados y ocupados por grupos sociales de mayor nivel socioeconómico.

De hecho en la ciudad se crearon espacios poblacionales diferentes, con una heterogeneidad que en la actualidad se mantiene, mostrando una combinación de subespacio que cumplieron y cumplen determinadas funciones como parte de la historia vivida por los procesos pasados y presentes. En consecuencia la ciudad se desarrolló sobre la base de cinco núcleos de población, destacándose los creados alrededor de la bahía (parte más antigua) y del río Almendares, sirviendo de base para el fomento de actividades industriales, propiciado por la disponibilidad del recurso hídrico, las que con el transcurso del tiempo se fueron convirtiendo en lugar de residencia de familias que disponían de muy pocos ingresos, una vez deteriorado el ambiente natural. Otra de las corrientes corresponde a la compactación de los espacios centrales, creación de diferentes espacios aislados surgidos en la segunda mitad de este siglo y por último, la absorción de los pueblos antiguos. Lo anterior define la forma irregular, de herradura o semidigital, que fuera referido en otras ocasiones por múltiples autores. Por otra parte fue el proceso de compactación lo que ha provocado en cierto modo la heterogeneidad interna de la ciudad, estando lo más homogéneo asociado a lo más antiguo, por cierto lo de menor extensión superficial (La Habana Colonial).

Resulta evidente que los determinantes esenciales de la heterogeneidad, están asociados en el caso que nos ocupa a la organización espacial del proceso de urbanización, donde el punto de vista histórico-evolutivo y segregacional son características del transcurso de este proceso esencialmente típico de una sociedad capitalista. No hay que olvidar que La Habana se constituyó básicamente en ese período (antes de 1959).

Por lo tanto, la influencia natural, aunque evidente, pierde importancia al observarse que en los contextos del fondo natural analizados, se desarrollan paisajes notablemente diferenciados desde el punto de vista social e infraestructural, que definen a su vez ambientes con marcado grado de heterogeneidad que se traducen en diversos grados de favorabilidad y deterioros (véase figura 3 ). 


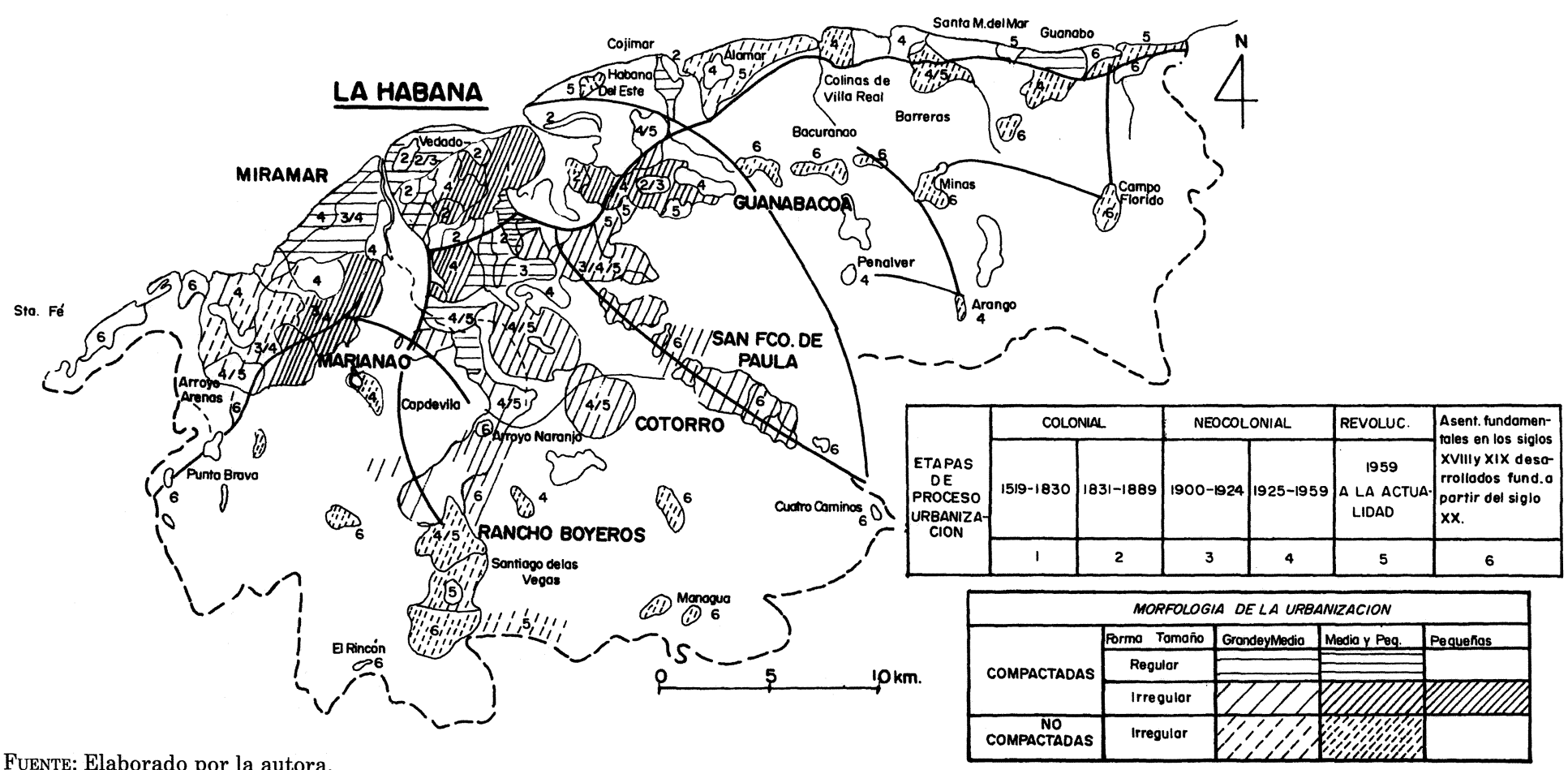

Figura 3.-Proceso de urbanización de ciudad de La Habana. 
Identificación y evaluación ambiental de La Habana

No se pretende en esta ocasión brindar una explicación completa de un problema polifacético dependiente de múltiples factores. La intención es aportar elementos de juicio objetivos, permitiendo discriminar una gama lo suficientemente amplia de variables e indicadores como para facilitar la descripción a un determinado nivel, utilizando una escala que permite establecer comparaciones entre los distintos espacios que integran la ciudad.

Resulta imposible además considerar un número mayor de variables e indicadores, ni mucho menos provocan una evaluación para los numerosos factores que determinan e influyen en el medio ambiente urbano. Por lo que sólo abordaremos un conjunto de aspectos, luego de la selección minuciosa del material disponible. En consecuencia asumimos las dimensiones siguientes:

- Aquellas asociadas a la temporalidad del proceso de urbanización, capaces de leer los contextos que diferencian tanto los modelos urbanísticos de cada etapa como la valoración del suelo urbano, especialmente referidos a las localizaciones sociales e industriales que en cierto sentido justifican el crecimiento del asentamiento.

- Las que son capaces de caracterizar el funcionamiento ambiental, principalmente físico, tomados como indicadores de los deterioros acumulados o generado en períodos recientes.

- Los que aunque con relativa independencia en el proceso de asimilación residencial, sirven de soporte natural para el desarrollo del fondo habitable e influyen con diferente intensidad en el actual funcionamiento ambiental.

En la identificación y evaluación ambiental según comportamiento estructuro-sustancial, fue empleado en conjunto de variables e indicadores, tomando como eje central las grandes etapas históricas, las condiciones naturales, así como el estado y condiciones de habitabilidad de la vivienda, estado técnico de la red de abasto y evacuación de residuales, calidad del aire y áreas verdes, complementaron la delimitación, dotando a las mismas de una cierta individualidad, 
fruto de lo cual es la marcada diferenciación que desde el punto de vista ambiental manifiesta la ciudad.

Por último y como vía de incorporación de elementos que informan acerca del fondo natural del territorio, en el funcionamiento y diferenciación ambiental actual, se procede establecer indicadores que valoren éstos, seleccionándose por su importancia, nivel de síntesis y de información las siguientes: localización en la llanura litoral o próxima a ella y localización en llanuras interiores.

Cabe destacar que esta localización es portadora de influencia en el funcionamiento mesoclimático (más brisa, menos lluvia), también de información acerca del escurrimiento por constituir esta llanura litoral una unidad con predominio de escurrimiento subterráneo, aunque en algunos sectores están cortados por ríos que forman valles profundos sin alta potencialidad a las inundaciones. En tanto el resto de las llanuras (interiores) muestran peligros sensibles, especialmente asociados con el régimen hidroclimático y orográfico.

Para la caracterización de la situación ambiental se parte de cada nivel obtenido (favorable, medianamente favorable, desfavorable y muy desfavorable, como aparece en la figura 4 y el cuadro I). A la escala de toda la ciudad se identifican ciento cuarenta y tres unidades ambientales, de ellas de $12,58 \%$ evaluadas con ambientes muy desfavorables, el $39,86 \%$ desfavorables, el $34,26 \%$ medianamente favorables y el 13,28\% como favorable.

Las unidades ambientales mejor evaluadas coinciden con los espacios urbanos creados en las tres últimas décadas del período capitalista, representando la expansión hacia el oeste de la ciudad y en menor medida al este de la bahía, por capas de mayor nivel socioeconómico, así como las ampliaciones realizadas en la etapa revolucionaria. Predominan las viviendas en buen estado con buenas condiciones de habitabilidad y casi todos los casos se ubican en la llanura costera.

Las unidades con evaluaciones ambientales más desfavorables coinciden en su mayoría con áreas urbanizadas durante la etapa colonial y primeras dos décadas de este siglo, las que han sufrido un proceso de deterioro progresivo, vinculado al deficiente mantenimiento que se les brindó a la infraestructura allí existente. En nivel semejan-

$$
-361-
$$




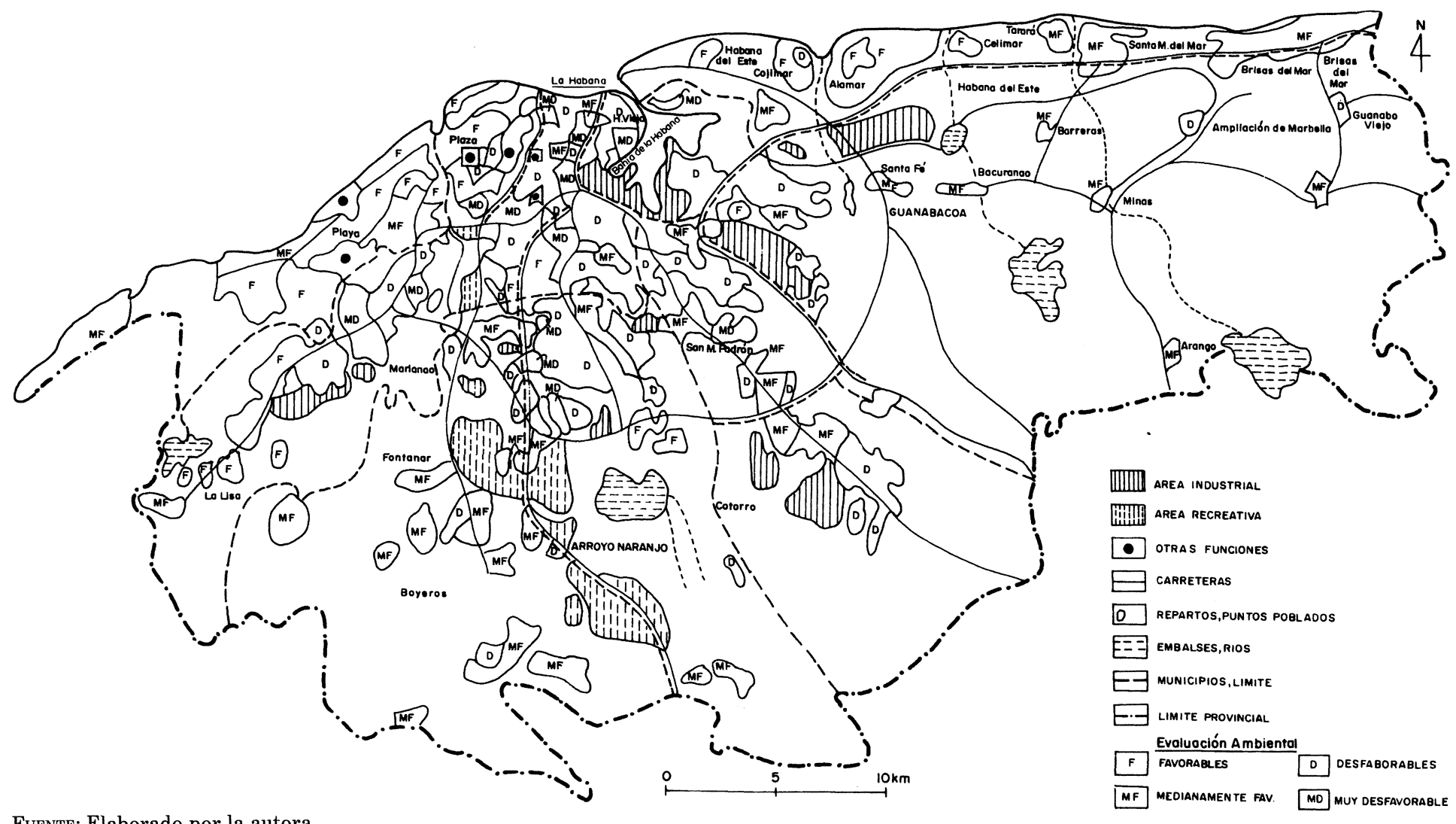

FuENTE: Elaborado por la autora.

Figura 4.-Esquema de evaluación ambiental en la ciudad de La Habana. 
CuAdro I

\begin{tabular}{|c|c|c|c|c|c|c|c|c|c|c|c|c|c|c|c|}
\hline \multirow{2}{*}{\multicolumn{2}{|c|}{$\begin{array}{l}\text { PROCESO DE } \\
\text { UREAMIZACION }\end{array}$}} & \multirow{2}{*}{\multicolumn{2}{|c|}{$\begin{array}{l}\text { DISTRIBUCION POR } \\
\text { PERIODOS HISTORICOS } \\
\text { DE U.A. }\end{array}$}} & \multirow{2}{*}{\multicolumn{4}{|c|}{$\begin{array}{l}\text { LOCALIZACION GEOQ. } \\
\text { U.A. POR PERIODOS } \\
\text { HISTORICOS }\end{array}$}} & \multicolumn{8}{|c|}{ DISTRIBUCION SEGUN NIVELES DE EVALACION AMBIENTAL } \\
\hline & & & & & & & & \multicolumn{2}{|c|}{ MUY DEsfavorable } & \multicolumn{2}{|c|}{ DESFAVORABLE } & \multicolumn{2}{|c|}{ MED. FAVORAGLE } & \multicolumn{2}{|c|}{ FAVORABLE } \\
\hline etapas & PERTOOS & No. & $\%$ & \begin{tabular}{|l} 
UANUR \\
LIT. \\
\end{tabular} & $\%$ & ". & $\%$ & no. & $\%$ & no. & $\%$ & No. & $\%$ & no. & $\%$ \\
\hline \multirow{3}{*}{$\begin{array}{l}\frac{1}{2} \\
\frac{a}{2} \\
0 \\
0 \\
0\end{array}$} & $H_{1}$ & • & 3,59 & 8 & 13,11 & - & - & 2 & 25 & 5 & 62,3 & 1 & 12,5 & - & - \\
\hline & $\mathrm{H}_{2}$ & 10 & 6,99 & $>$ & 0,53 & 3 & 4,91 & 4 & 40 & 4 & 40 & 2 & 20 & - & - \\
\hline & $\mathrm{H}_{2}-3$ & 10 & 6,09 & 8 & 13,33 & 2 & 2,40 & 1 & 10 & 3 & 30 & 4 & 40 & 2 & 20 \\
\hline \multirow{4}{*}{ 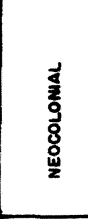 } & $\mathrm{H}_{3}$ & 2 & 0,29 & 1 & 1,63 & 8 & 0.75 & 1 & 11,11 & 5 & 55,5 & 2 & 22,2 & 1 & 11,11 \\
\hline & $\mathrm{H}_{2}-3-4$ & I & 4,80 & 7 & 10,47 & - & - & 2 & 20,87 & 3 & 71,42 & - & - & - & - \\
\hline & $\mathrm{H}_{3}-4$ & 7 & 4,89 & 7 & 11,47 & - & - & - & - & - & - & 3 & 42,85 & 4 & 87,14 \\
\hline & $\mathrm{H}_{4}$ & 20 & 19,50 & 13 & 21,66 & 15 & 10,07 & 3 & 10,71 & 10 & 35,71 & - & 28,57 & 7 & 28 \\
\hline \multirow{5}{*}{ 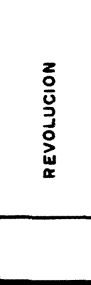 } & $\mathrm{H}_{4}-3$ & 31 & 21,67 & 3 & 4,21 & 28 & 34,14 & 4 & 12,90 & 12 & 38,70 & 13 & 41,83 & 2 & 8,48 \\
\hline & $\mathrm{H}_{5}$ & 10 & 0,00 & 5 & 8,18 & 5 & 8,08 & - & - & 2 & 20 & $:$ & 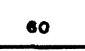 & 2 & 20 \\
\hline & $\mathrm{H}_{5}{ }^{4-5}$ & 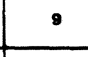 & 8,29 & 2 & 3,27 & 7 & 0,5 & 1 & 11,11 & - & 66,86 & 2 & 22,22 & 2 & - \\
\hline & $\mathrm{H}_{\mathrm{B}}$ & 14 & 9,79 & 4 & 0,58 & 10 & 2,19 & - & - & 4 & 28,57 & • & 64, 28 & 1 & 7,14 \\
\hline & TOTAL & 143 & 100 & 60 & 100 & 83 & 100 & 10 & 12,50 & 57 & 30,00 & AS & 34,28 & 10 & 13,28 \\
\hline
\end{tabular}


te se sitúan otros que a pesar de ser de más reciente asimilación (hasta 1959), no contaron con un plan de regulación urbanística adecuado, siendo determinante en muchos casos el bajo potencial constructivo de las áreas seleccionadas, favoreciendo el arribo de familias de bajos ingresos, obligados, por problemas económicos a alojarse en viviendas con precarias condiciones para el hábitat. De hecho, el análisis de los deterioros físicos y sociales del ambiente, muestran cierta coincidencia en la ubicación de las unidades ambientales a partir del casco histórico y en dirección sur, sureste y suroeste, siguiendo los principales ejes viales coincidiendo en algunos casos con núcleos más antiguos de población.

El nivel definido como medianamente favorable contiene a las unidades ambientales con un predominio de viviendas en estado bueno y regular, existiendo en algunos problemas con la habitabilidad, suministro de agua y evacuación de residuales. Éstas están distribuidas en casi todos los períodos históricos, aunque se presenta una ligera concentración es el correspondiente a la segunda mitad de este siglo. De igual forma se comporta el indicador posición geográfica, ya que no hay cambios notables en la ubicación de las mismas en uno $u$ otro tipo de llanura.

Hay que decir que la situación ambiental de la Ciudad de La Habana es fruto de un proceso de urbanización heterogéneo, resultado de la diferenciación de las condiciones naturales y de la segregación social que acompañan sus principales etapas de crecimiento.

\section{BIBLIOGRAFÍA}

Azevedo, L. G. (1988): Ensayo metodológico de identificación y evaluación de unidades ambientales. La estación ecológica de PIRAPITINGA. Brasil, $20 \mathrm{pp}$.

BARTKowsKI, T. (1979): Selected problems of urban Ecology, Varsovia, 191 pp.

BaRRIOS, P. (1988): Espacios rurales y urbanos en áreas industrializadas. Madrid, 252 pp.

Cabañas, L. (1989): Sistema de protección del medio ambiente de la provincia Ciudad de La Habana. Bases conceptuales, 31 pp. (mimeografiado).

CCE (1990): Libro Verde sobre el medio ambiente urbano. Bruselas, 81 pp. NC 9300006187.

Estado del medio ambiente. Criterios para su evaluación. La Habana. Comité Estatal de Normalización. 
Dominguez, B. P.: El medio ambiente. Un ensayo integrado desde distintos puntos de vista. La problemática del medio ambiente urbano. Córdoba, España, $183 \mathrm{pp}$.

DPPFA (1990): Esquema del Plan Director del año 2010. Ciudad de La Habana. La Habana, $144 \mathrm{pp}$.

Fernández, J. M. (1988): Aspectos más relevantes del desarrollo urbano de la ciudad de La Habana (1519-1987).

GIAComin, V. (1981): Hacia un urbanismo humanizado. El Correo de la Unesco. París, 23 pp.

GonzÁlez, M. (1990): «La Habana es y no es». Conferencia presentada en el IV Congreso Iberoamericano de Urbanismo. La Habana.

GutiÉRREZ DE MAC GREGor, M. (1994): Geografia urbana. El nacimiento de las primeras ciudades del viejo mundo, Facultad de Arquitectura e Instituto de Geografía, UNAM, $112 \mathrm{pp}$.

IÑıGUEz, L. (1990): Los enfoques ecológicos integrales en las investigaciones de los asentamientos poblacionales. IPF La Habana, 2 pp (mimeografiado).

LEUChSEN, E. RoIg DE (1938): Historia de La Habana, La Habana.

Marrero, L. (1951): Geografia de Cuba, La Habana, 472 pp.

PRiETo, C. J. (1984): La generalización en el mapa de paisajes de La Habana y Ciudad de La Habana. Facultad de Geografía Univrsidad de La Habana. Trabajo de curso, 17 pp. (inédito).

PuYol, R. (1989): Geografía Humana, Madrid, 578 pp.

Pérez, N. (1995): La diferenciación del ambiente urbano en Ciudad de La Habana. Potencialidades para la gestión comunitaria. Tesis de doctorado. Universidad de La Habana, $113 \mathrm{pp}$.

REY, G. (1991): La Habana y la crisis urbana, desafios y soluciones. Grupo de Desarrollo de la Ciudad, 8 pp.

La sociedad y el medio ambiente. Concepción de los científicos sométricos. Edit. Progreso, Moscú, 1981, 235 pp.

RESUMEN: La diferenciación ambiental en una ciudad obedece a una serie de factores que de conjunto le confieren carácter sistémico. La Ciudad de La Habana se presenta como un espacio constituido mediante un largo y complicado proceso de urbanización, donde el componente natural sin ser determinante, potenció la presencia de un medio ambiente complejo y diferenciado.

Palabras Claves: Heterogeneidad ambiental. Medio ambiente. Ciudad de La Habana. Unidad ambiental. 